\section{Theoretical \& Applied Science}

p-ISSN: 2308-4944 (print) e-ISSN: 2409-0085 (online)

Year: 2018 Issue: $06 \quad$ Volume: 62

Published: $30.06 .2018 \quad$ http://T-Science.org
Indira Adil Safarova

Doctoral student of Oncology Department of Azerbaijan Medical University

Abuzer Yusif Kaziev

Professor, Doctor of Medical Sciences, Department of Oncology, Azerbaijan Medical University

Gulnara Alisa Jafarova

Candidate of Biological Sciences, doctor-laboratory assistant of the Oncological Clinic Azerbaijan Medical

University

Ismail Adil Gafarov

c.ph.-m.n. Art. teachers.

Department of Medical Physics and Informatics

Azerbaijan Medical University

\title{
STUDY OF INFORMATIVE VALUE AND PROGNOSTIC SIGNIFICANCE OF SOME CYTOKINES AND ANTIMICROBIAL PEPTIDES FOR EARLY DETECTION OF METASTASES IN PATIENTS WITH CERVICAL CANCER
}

\begin{abstract}
The article presents the results of a study conducted to study the informative value and prognostic significance of some cytokines and antimicrobial peptides in the early detection of metastases in patients with cervical cancer. For this purpose, in blood in 46 patients without lymph node metastases was determined the content of some cytokines (IL-2, IL-6, IL-10 and TNF- $\alpha$ ) and antimicrobial peptides (endothelium, L-FABP, endotoxin, lactoferrin) by an enzyme immunoassay analysis. To determine the informativeness and prognostic significance of the studied indicators, was used a statistical dispersion of test ANOVA and ROC analysis. The results of the study showed a significant increase in proinflammatory cytokines and antimicrobial peptides in the serum of patients with cervical cancer. Elevated values were observed in those patients in whom various metastases to the lymph nodes were observed during the course of 6 months to 3 years. It has been established that IL-2, TNF$\alpha, I L-6$, endothelin and L-FABP have prognostic and diagnostic significance for the early detection of metastases in patients with cervical cancer.
\end{abstract} protein)

Key words: cervical cancer, cytokines, tumor necrosis factor, endothelium, L-FABP (fatty acid binding

\section{Language: Russian}

Citation: Safarova IA, Kaziev AY, Jafarova GA, Gafarov IA (2018) STUDY OF INFORMATIVE VALUE AND PROGNOSTIC SIGNIFICANCE OF SOME CYTOKINES AND ANTIMICROBIAL PEPTIDES FOR EARLY DETECTION OF METASTASES IN PATIENTS WITH CERVICAL CANCER. ISJ Theoretical \& Applied Science, 06 (62): 201-206.

Soi: http://s-o-i.org/1.1/TAS-06-62-35 Doi: crostef https://dx.doi.org/10.15863/TAS.2018.06.62.35

\section{ИЗУЧЕНИЕ ИНФОРМАТИВНОСТИ И ДИАГНОСТИЧЕСКОЙ ЗНАЧИМОСТИ НЕКОТОРЫХ ЦИТОКИНОВ И АНТИМИКРОБНЫХ ПЕПТИДОВ ДЛЯ РАННЕГО ВЫЯВЛЕНИЯ МЕТАСТАЗОВ У БОЛЬНЫХ С РАКОМ ШЕЙКИ МАТКИ}

Аннотация: В статье представлены результаты исследования, проведенного с иелью изучения информативности и прогностической значимости некоторых цитокинов и антимикробных пептидов при раннем выявлении метастазов у больных раком шейки матки РШМ. С этой целью в крови у 46 больных без метастазов на лимфоузль, определяли содержание некоторых циитокинов (ИЛ-2, ИЛ-6, ИЛ-10 и ФНО- $\alpha$ ) и антимикробных пептидов (ендотелин, L-FABP, эндотоксин, лактоферрин) путем иммуноферментного анализа. Для определения информативности и прогностической значимости изучаемых показателей использовали статистический дисперс тест-ANOVA и ROC анализ. Результаты исследования показали значительное повышение провоспалительных цичтокинов и антимикробных пептидов в сыворотке крови больных РШМ. Повышенные значение наблюдались у тех больных в которых в течения от 6 месячев до 3 лет наблюдалось различные метастазы в лимфоузль. Установлено, ИЛ-2, ФНО-, ИЛ-6, ендотелина и L- 
FАВР имеют прогностическое и диагностическое значение для раннего выявления метастазов у больных РШМ.

Ключевые слова: рак шейки матки, цитокины, фактор некроза опухоли, ендотелин, L-FABP (белок связывающие жирные кислоты)

\section{Введение}

В последнее время активно изучаются воспалительные и регуляторные механизмы, участвующие в возникновении и прогрессировании РШМ. Особое внимание в связи с этим уделяется дисбалансу клеточных медиаторов, которые регулируют функциональную активность клеток, их пролиферацию и апоптоз. Медиаторами межклеточной взаимодействия и иммунорегуляции являются цитокины, среди которых ключевую роль в развитии иммунного ответа играют фактор некроза опухоли-альфа (ФНО-а), ИЛ-2 и ИЛ-6 [1, $3,7,15]$.

Многие авторы подчёркивают роль цитокинов и антимикробных пептидов (АМП) в развитии рака шейки матки (РШМ). Высокий уровень провоспалительных цитокинов и АМП у большинства больных с метастазами РШМ подтверждает их участие в прогрессировании поздних осложнений.

Имеются много фактов, свидетельствующих о том, что некоторые медиаторы иммунной системы стимулирует опухолевую прогрессию. В последнее время считают, что в процессе возникновения и развития опухоли происходит нарушение баланса между секрецией цитокинов (ИЛ-2, ИЛ-6, ИЛ-10 и ФНО) и АМП (эндотоксин, лактоферрин) $[1,2,7,16]$.

Полученные результаты исследования могут использоваться для ранней неинвазивной диагностики и оценки процессов, протекающих при РШМ и прогнозирования метастазов.

Определение факторов, определяющих темпы развития РШМ, и выявление новых диагностических маркеров раннего выявления метастазов являются актуальной задачи онкологии. Решение этой проблемы имеет принципиальное значение для оптимизации подходов к ранней диагностике и профилактике РШМ.

Целю данной работы, является изучение информативности и диагностических значений некоторых цитокинов и АМП при прогнозировании метастазов у больных РШМ.

Материал и методы. С этой целью было обследовано венозная кровь 46 больных с РШМ, находившихся на лечении в онкологической клинике Азербайджанского Медицинского Университета. В течения от 6-ти мес. до 3 лет после поступления в клинику у 13 из них выявлено различные метастазы в лимфоузлы. Кровь больных была взята при поступлении больных в клинику. Диагноз был поставлен на основании материала биопсии.

Для исследования содержания ИЛ-2, ИЛ-6, ИЛ-10, ФНО- $\alpha$, эндотелина, эндотоксина, лактоферрина и белка, связывающего жирные кислоты (L-FABP) в сыворотке крови использовали набор реагентов производства «Вектор-Бест» (Россия). Статистическую обработку результатов проводили методами вариационного, дисперсионного и ROC-анализов с помощью программ EXCEL-2013 и SPSS-20 $[5,6]$.

Результаты и обсуждения. В целях определения прогностической значимости изученных лабораторных показателей для выявления метастазов больных распредели на 2 группы: I группа, у которых не выявлены метастазы в течении 3-х лет после операции (n=33), II группа, у которых выявлены различные метастазы в лимфоузлы в течении от 6-ти мес. до 3-х лет после лечения ( $\mathrm{n}=33)$.

C помощью статистической обработки результатов получены следующие результаты. Результаты дисперсионного анализа представлены в табл. 1 .

Таблица 1

Дисперсионный анализ изученных показателей у больных РШМ

\begin{tabular}{|c|c|c|c|c|c|c|c|c|c|}
\hline \multicolumn{2}{|l|}{ Показатели } & $\mathbf{N}$ & Среднее & $\begin{array}{l}\text { Стан. } \\
\text { откл. }\end{array}$ & $\begin{array}{l}\text { Стан. } \\
\text { ошиб. }\end{array}$ & $\begin{array}{l}95 \% \\
\text { НГр }\end{array}$ & $\begin{array}{l}95 \% \\
\text { Вгр }\end{array}$ & $\mathbf{F}$ & $\mathbf{p}$ \\
\hline \multirow{2}{*}{ L-FABP, нг/мЛ } & MC (-) & 33 & 1,83 & 1,43 & 0,25 & 1,32 & 2,34 & 5,026 & 0,030 \\
\hline & $\mathrm{MC}(+)$ & 13 & 2,98 & 1,88 & 0,52 & 1,85 & 4,12 & & \\
\hline \multirow{2}{*}{ Эндотелин, пг/мл, } & $\mathrm{MC} \mathrm{(-)}$ & 33 & 8,50 & 0,68 & 0,12 & 8,26 & 8,75 & 9,111 & 0,004 \\
\hline & $\mathrm{MC} \mathrm{(+)}$ & 13 & 9,19 & 0,74 & 0,20 & 8,75 & 9,64 & & \\
\hline \multirow{2}{*}{ Эндотоксин, пг/мл } & $\mathrm{MC}(-)$ & 33 & 0,161 & 0,054 & 0,009 & 0,142 & 0,180 & 3,139 & 0,083 \\
\hline & $\mathrm{MC} \mathrm{(+)}$ & 13 & 0,192 & 0,050 & 0,014 & 0,161 & 0,222 & & \\
\hline \multirow{2}{*}{ Лактоферрин, мкг/г } & MC (-) & 33 & 3,73 & 1,07 & 0,19 & 3,35 & 4,11 & 0,005 & 0,944 \\
\hline & $\mathrm{MC}(+)$ & 13 & 3,70 & 1,12 & 0,31 & 3,03 & 4,38 & & \\
\hline
\end{tabular}




\section{Impact Factor:}

\begin{tabular}{lr|lr} 
ISRA $($ India & $=\mathbf{1 . 3 4 4}$ & SIS $($ USA) & $=\mathbf{0 . 9 1 2}$ \\
ISI $($ Dubai, UAE) $=\mathbf{0 . 8 2 9}$ & PИНЦ (Russia) $=\mathbf{0 . 2 0 7}$ \\
GIF (Australia) $=\mathbf{0 . 5 6 4}$ & ESJI (KZ) & $=\mathbf{4 . 1 0 2}$ \\
JIF & $=\mathbf{1 . 5 0 0}$ & SJIF $($ Morocco $)=\mathbf{2 . 0 3 1}$
\end{tabular}

\begin{tabular}{|c|c|c|c|c|c|c|c|c|c|}
\hline \multirow{2}{*}{ TNF- $\alpha$, пг/мл } & MC (-) & 33 & 19,2 & 11,1 & 1,9 & 15,3 & 23,2 & 4,648 & 0,037 \\
\hline & $\mathrm{MC} \mathrm{(+)}$ & 13 & 27,0 & 10,8 & 3,0 & 20,5 & 33,5 & & \\
\hline \multirow{2}{*}{ Il-10, пг/мл } & MC (-) & 33 & 14,3 & 3,7 & 0,6 & 13,0 & 15,6 & 0,134 & 0,716 \\
\hline & $\mathrm{MC} \mathrm{(+)}$ & 13 & 14,8 & 4,5 & 1,2 & 12,1 & 17,4 & & \\
\hline \multirow{2}{*}{ Il-6, пг/мл } & MC (-) & 33 & 6,04 & 1,79 & 0,31 & 5,40 & 6,67 & 7,225 & 0,010 \\
\hline & $\mathrm{MC} \mathrm{(+)}$ & 13 & 7,71 & 2,15 & 0,60 & 6,41 & 9,01 & & \\
\hline \multirow{2}{*}{ Il-2, пг/мл } & MC (-) & 33 & 28,2 & 12,8 & 2,2 & 23,7 & 32,7 & 7,703 & 0,008 \\
\hline & $\mathrm{MC}(+)$ & 13 & 40,9 & 16,8 & 4,7 & 30,8 & 51.1 & & \\
\hline
\end{tabular}

Прим: МС(-) - больные РШМ без метастазов, МС(+) - больные РШМ с метастазами.

У больных, которых не в дальнейшем не развились метастазы средняя величина ИЛ-2 составила 28,2 $\pm 2,2$ пг/мл; ИЛ-6 - 6,04 $\pm 0,31$ пг/мл; ФНО- $\alpha-19,2 \pm 1,9$ пг/мл. Полученные результаты показали, что у больных без метастазов уровень цитокинов значительно ниже по сравнению с данными больных с метастазами. У больных с метастазами среднее величина ИЛ-2 составила $40,9 \pm 4,7$ пг/мл (p=0,008); ИЛ-6 - 7,71 $\pm 0,60$ пг/мЛ $(\mathrm{p}=0,010) ;$ ФНО- $\alpha 27,0 \pm 3,0$ пг/мл $(\mathrm{p}=0,037)$.

Среднее значение эндотелина у больных без дальнейших метастазов составила $8,50 \pm 0,12$
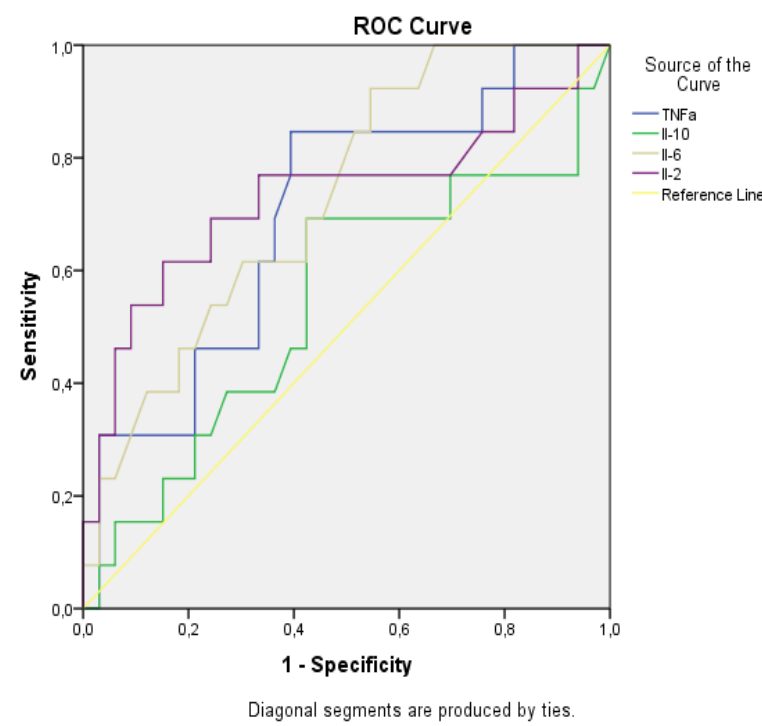

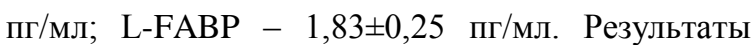
анализа выявили достоверное увеличение некоторых АМП у больных РШМ с выявленными метастазами. Как видно из полученных результатов, у больных с метастазами, уровень эндотелина составила $9,19 \pm 0,20$ пг/мл $(\mathrm{p}=0,004)$; L-FABP $-2,98 \pm 0,52$ пг/мл $(\mathrm{p}=0,030)$.

C помощью ROC-анализа определяли специфичность и чувствительность цитокинов и АМП при раннем выявлении метастазов у больных РШМ (рис. 1).

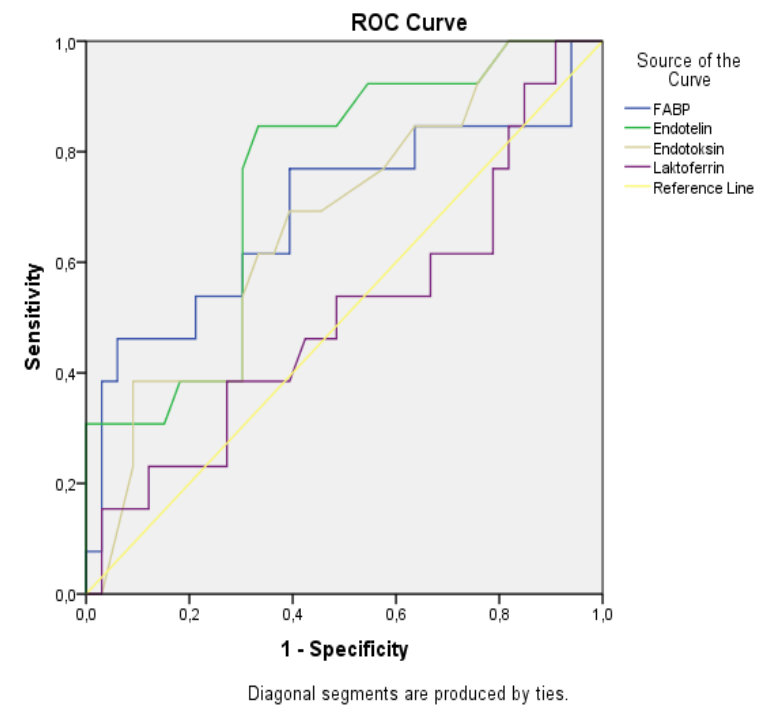

\begin{tabular}{|c|r|r|r|r|r|}
\hline Изменчивость теста & \multirow{2}{*}{\begin{tabular}{c}
\multirow{2}{*}{ Пов $)$} \\
Площадь
\end{tabular}} & \multirow{2}{*}{ Станд. откл. } & \multirow{2}{*}{ Р дост. } & \multicolumn{2}{|c|}{ 95\% доверительный интервал } \\
\cline { 5 - 6 } & & & Ниж. пред. & Верх. пред. \\
\hline ИНО-а & 0,698 & 0,085 & 0,038 & 0,531 & 0,865 \\
\hline ИЛ-10 & 0,544 & 0,101 & 0,643 & 0,346 & 0,742 \\
\hline ИЛ-6 & 0,728 & 0,078 & 0,017 & 0,576 & 0,881 \\
\hline ИЛ-2 & 0,732 & 0,096 & 0,015 & 0,545 & 0,919 \\
\hline L-FАВР & 0,692 & 0,098 & 0,044 & 0,500 & 0,884 \\
\hline Эндотелин & 0,746 & 0,078 & 0,010 & 0,592 & 0,900 \\
\hline Эндотоксин & 0,668 & 0,087 & 0,079 & 0,498 & 0,838 \\
\hline Лактоферрин & 0,505 & 0,100 & 0,961 & 0,309 & 0,700 \\
\hline
\end{tabular}

Рисунок 1. ROC-графики цитокинов и АМП при раннем выявлении метастазов у больных РШМ 
На основании ROC-анализа можно сделать вывод, что специфичность и чувствительность тестов ИЛ-2, ИЛ-6 и ФНО- $\alpha$, а также L-FABP и эндотелина в прогнозировании метастазов у больных являются статистически значимыми.

Далее определили наиболее удаленные точки от опорной линии - точки отсечения (cut of point) в координатах ROC-кривых, где суммарное значение специфичности и чувствительности являются наибольшей. По этим точкам вычисляли специфичность и чувствительность каждого теста (табл. 2).

Специфичность и чувствительность изученных показателей в точках отсечений

Табл. 2

\begin{tabular}{|c|c|c|c|c|c|c|c|c|}
\hline Стат. & L-FАВР & $\begin{array}{c}\text { Эндотел } \\
\text { ин }\end{array}$ & $\begin{array}{c}\text { Эндоток } \\
\text { син }\end{array}$ & $\begin{array}{c}\text { Лактофе } \\
\text { ррин }\end{array}$ & ФНО-а & ИЛ-10 & ИЛ-6 & ИЛ-2 \\
\hline $\begin{array}{c}\text { Точка } \\
\text { отсечения }\end{array}$ & 3,8 & 8,8 & 0,18 & 5 & 19,5 & 14,6 & 5,4 & 44 \\
\hline МС (+) & 13 & 13 & 13 & 13 & 13 & 13 & 13 & 13 \\
\hline++ & 6 & 10 & 8 & 2 & 11 & 9 & 12 & 8 \\
\hline Чв & 46,2 & 76,9 & 61,5 & 15,4 & 84,6 & 69,2 & 92,3 & 61,5 \\
\hline $\pm m p$ & 13,8 & 11,7 & 13,5 & 10,0 & 10,0 & 12,8 & 7,4 & 13,5 \\
\hline MC (-) & 33 & 33 & 33 & 33 & 33 & 33 & 33 & 33 \\
\hline-- & 31 & 23 & 22 & 32 & 20 & 19 & 15 & 28 \\
\hline Сп & 93,9 & 69,7 & 66,7 & 97,0 & 60,6 & 57,6 & 45,5 & 84,8 \\
\hline $\pm m p$ & 4,2 & 8,0 & 8,2 & 3,0 & 8,5 & 8,6 & 8,7 & 6,2 \\
\hline OДЦ & 37 & 33 & 30 & 34 & 31 & 28 & 27 & 36 \\
\hline$\%$ & 69,8 & 62,3 & 56,6 & 64,2 & 58,5 & 52,8 & 50,9 & 67,9 \\
\hline $\pm m p$ & 6,3 & 6,7 & 6,8 & 6,6 & 6,8 & 6,9 & 6,9 & 6,4 \\
\hline pPV & 75,0 & 50,0 & 42,1 & 66,7 & 45,8 & 39,1 & 40,0 & 61,5 \\
\hline $\pm m p$ & 15,3 & 11,2 & 11,3 & 27,2 & 10,2 & 10,2 & 8,9 & 13,5 \\
\hline nPV & 81,6 & 88,5 & 81,5 & 74,4 & 90,9 & 82,6 & 93,8 & 84,8 \\
\hline $\pm m p$ & 6,3 & 6,3 & 7,5 & 6,7 & 6,1 & 7,9 & 6,1 & 6,2 \\
\hline LR+ & 7,62 & 2,54 & 1,85 & 5,08 & 2,15 & 1,63 & 1,69 & 4,06 \\
\hline & хор. & поср. & не пр. & хор & поср. & не пр. & не пр. & поср. \\
\hline LR- & 0,57 & 0,33 & 0,58 & 0,87 & 0,25 & 0,53 & 0,17 & 0,45 \\
\hline & не пр & поср. & не пр & не пр & поср. & не пр & хор. & поср. \\
\hline
\end{tabular}

Прим: Чв - чувствительность; Сn - Специфичность; ОДЦ - общая диагностическая ценность; ++ (-) - истинно-положительные (отрицательные) результаты; $\pm m p-95 \%$ доверительный интервал полученных результатов; рPV (nPV) - предсказательная полезность положительного (отрицательного) результата; LR+ (LR-) - отношения правдоподобия положительного (отрицательного) результата.

Для определения силы фактора в прогнозировании метастазов у больных РШМ после лечения использован дисперс-тест по методу Снедекора. Результаты теста показали, что повышение содержание ИЛ-2 выше чем 44 пг/мл (СФ - 27,4\%; p < 0,001), ИЛ-6 $\geq 5,4$ пг/мЛ $(\mathrm{C} \Phi-14,6 \%, \mathrm{p}=0,004) ;$ ФНО- $\alpha \geq 19,5$ пг/мл (СФ
$-19,9 \%, \mathrm{p}=0,001) ; \mathrm{L}-\mathrm{FABP}-\mathrm{a} \geq 3,8$ нг/мл (CФ$29,3 \%, ; \mathrm{p}<0,001)$ и эндотелина $\geq 8,8$ пг/мл (СФ $21,8 \% ; \quad \mathrm{p}<0,001)$ является достоверными критериями в прогнозировании прогрессировании метастазов у больных РШМ (таблица 3). 


\begin{tabular}{l|lr|ll|ll} 
& ISRA (India) & $=\mathbf{1 . 3 4 4}$ & SIS (USA) & $=\mathbf{0 . 9 1 2}$ & ICV (Poland) & $=\mathbf{6 . 6 3 0}$ \\
Impact Factor: & ISI (Dubai, UAE) $=\mathbf{0 . 8 2 9}$ & PUHЦ (Russia) $=\mathbf{0 . 2 0 7}$ & PIF (India) & $=\mathbf{1 . 9 4 0}$ \\
& GIF (Australia) & $\mathbf{0 . 5 6 4}$ & ESJI (KZ) & $=4.102$ & IBI (India) & $=\mathbf{4 . 2 6 0}$ \\
& JIF & $=\mathbf{1 . 5 0 0}$ & SJIF (Morocco) & $=\mathbf{2 . 0 3 1}$ & & \\
\hline
\end{tabular}

Дисперсионный тест-ANOVA

Таблица 3 некоторых цитокинов и АМП у больных РШМ

\begin{tabular}{|c|c|c|c|c|}
\hline \multicolumn{2}{|c|}{ Пределы показателей } & $\begin{array}{c}\text { Сила фактора } \\
\mathbf{9 5 \%} \text { ДИ: ВГр-НГр) }\end{array}$ & F & p \\
\hline ИЛ-2 & $\geq 44,0$ & $\begin{array}{c}27,4 \% \\
(20,7-34,1)\end{array}$ & 16,6 & $<0,001$ \\
\hline ИЛ-6 & $\geq 5,4$ & $\begin{array}{c}14,6 \% \\
(6,7-22,5)\end{array}$ & 7,5 & 0,004 \\
\hline ИЛ-10 & $\geq 14,6$ & $\begin{array}{c}6,2 \% \\
(0,0-14,8)\end{array}$ & 2,9 & 0,055 \\
\hline ФНО- $\alpha$ & $\geq 19,5$ & $\begin{array}{c}19,9 \% \\
(12,5-27,3)\end{array}$ & 10,9 & $<001$ \\
\hline Эндотелин & $\geq 8,8$ & $\begin{array}{c}21,8 \% \\
(14,6-29,1)\end{array}$ & 12,3 & $<0,001$ \\
\hline L-ҒАВР & $\geq 3,8$ & $\begin{array}{c}29,3 \% \\
(22,8-35,9)\end{array}$ & 18,3 & 0,001 \\
\hline Эндотоксин & $\geq 0,18$ & $\begin{array}{c}7,1 \% \\
(0,0-15,7)\end{array}$ & 3,4 & 0,073 \\
\hline Лактоферрин & $\geq 5,0$ & $\begin{array}{c}5,3 \% \\
(0,0-14,1)\end{array}$ & 2,5 & $<01$ \\
\hline
\end{tabular}

ИЛ-2 синтезируемый Т-хелперными лимфоцитами, играет исключительно важную роль в дифференцировке и пролиферации Тклеток, принимает непосредственное участие в реализации механизмов противоопухолевой защиты и является активным стимулятором продукции ФНО- $\alpha$ [4].

ИЛ-6 является провоспалительным цитокином, иммуномодулятором, стимулируюет синтез других цитокинов и может выступать в роли индуктора канцерогенеза. ИЛ-6 обладает и мощным ангиогенным действием, стимулируя продукцию VEGF опухолевых клеток, и поэтому повышенная экспрессия IL-6, является фактором метастаза РШМ [10, 20].

ИЛ-10 относится к группе противовоспалительных цитокинов и основная функция ИЛ-10 состоит в угнетении синтеза цитокинов Th1-клетками и в снижении активности макрофагов, в том числе продукции провоспалительных цитокинов. Также полагают, что ИЛ-10 способствует стимуляции опухолевого роста в результате ингибирования Т-клеточного иммунного ответа $[17,19]$.

ФНО- $\alpha$ усиливает синтез других цитокинов и экспрессию молекул адгезии. Повышение концентрации ФНО-а приводит снижению противоопухолевого эффекта и повышению резистентности организма против развития опухолевого процесса [8].

Как видно из результатов, высокое содержание эндотоксина сопровождается усиленной продукцией провоспалительных цитокинов $[11,13]$. Лактоферрин в основном локализован во специфических гранулах полиморфноядерных нейтрофилов. является важной составляющей иммунной системы и иммуномодулирующими свойствами [14]. LFABP - связывает жирные кислоты и обеспечивает их интрацеллюлярную транспорт $[12,18]$. Эндотелин вызывает увеличение количества Т-лимфоцитов в тканях и органах, что в свою очередь привлекает другие иммунокомпетентные клетки, в частности макрофаги, которые продуцируют факторы, стимулирующие воспаление и деструкцию очага, что приводит к неблагоприятному прогнозу для нормальной репарации [9].

Полученные результаты свидетельствует о взаимосвязи повышения уровня L-FABP и эндотелина со степенью повреждения и метастазирования опухолевых клеток. Поэтому L-FABP и эндотелин являются чувствительными и специфичными маркерами повреждения ткани.

В связи с этим исследование уровня ИЛ-2, ФНО- $\alpha$, ИЛ-6, эндотелина и L-FABP может быть рекомендовано для оценки вероятности развития метастазирование опухолевых клеток у больных РШМ и может иметь прогностическое и диагностическое значение для раннего выявления метастазов. 


\begin{tabular}{l|lr|ll|ll} 
& ISRA (India) & $=\mathbf{1 . 3 4 4}$ & SIS (USA) & $=\mathbf{0 . 9 1 2}$ & ICV (Poland) & $=\mathbf{6 . 6 3 0}$ \\
Impact Factor: & ISI (Dubai, UAE) $=\mathbf{0 . 8 2 9}$ & PUHL (Russia) $=\mathbf{0 . 2 0 7}$ & PIF (India) & $=\mathbf{1 . 9 4 0}$ \\
& GIIF (Australia) & $=\mathbf{0 . 5 6 4}$ & ESJI (KZ) & $=4.102$ & IBI (India) & $=\mathbf{4 . 2 6 0}$ \\
& JIF & $=\mathbf{1 . 5 0 0}$ & SJIF (Morocco) & $=2.031$ & & \\
\hline
\end{tabular}

\section{References:}

1. Abakumova T.V., Antoneeva I.I., Qening T.P., Dolqova D.R., Qeninq C.O., Voronova O.C., Volqina I.V. (2013) Funksionalnoe sostoyaniye mikrofaqositov perifericeskoq krovi i spektr produsiruemix imi sitokinov pri rake sheyki matki// Ulyanovskiy mediko-bioloqiceskiy jurnal, №1, p.56-63.

2. Alyoshina Q.M., Kokryakov V.N., Shamova O.V., Orlov D.C. et al. (2010) Sovremennaya konsepsiya ob antimikrobnix peptidax kak molekulyarnix faktorax immuniteta // Medisinskiy akademiceskiy jurnal, №4, p.149160 .

3. Berezhnaya N.M.(2000) Interleykiny i formirovanie immunologicheskogo otveta pri zlokachestvennom rost // Allerg. i immunol. T. 1,№1.-p. 45-61.

4. Berezhnaya N.M., Goretskiy B.A. (1992) Interleykin-2 i zlokachestvennye obrazovaniya // Kiev: Naukova dumka, 172 p.

5. Dolzhnikov V., Stuchenkov A. (2008) Excel // SPb, BKhB- Peterburg,544 p.

6. Petri A. Sebin K. (2009) Nakladnaya statistika v meditsine // Perov. s. angl. V.P. Leonova M.: Geotar-Med, 168 p.

7. Simbirsev A.C. (2014) Sitokin;-novaya sistema requlyaisyii zashitnix reaksiy orqanizma// Sitokini i vospoleniye, t.2002, №1, p.32.

8. Chu W.M. (2013) Tumor necrosis factor // Cancer Lett., v. 328(2), p. 222-225.

9. Fathy Anan, Aziza E. Abdelrahman. (2018) EZH2, Endothelin-1, and CD34 as Biomarkers of Aggressive Cervical Squamous Cell Carcinoma: An Immunohistochemical Study// Turkish Journal of Pathology, №34, p.150-157.

10. Heikkila, Ebrahim S, Lawlor D.A. (2008) Systematic review of the association between circulating interleukin-6 (IL-6) and cancer. Eur J Cancer, №44, p. 937-45
11. Heine H, Rietschel ET, Ulmer AJ. The biology of endotoxin //Mol Biotechnol, 2001, No 19(3), p.279-296.

12. Huang H, McIntosh AL, Martin GG, Landrock KK, Landrock D, Gupta S, Atshaves BP, Kier AB, Schroeder F. (2014) Structural and functional interaction of fatty acids with human liver fatty acid-binding protein (L-FABP) T94A variant// FEBS J., №281, p.2266-2283.

13. Jessica I. Lundin; Harvey Checkoway (2010) Endotoxin and cancer//Ciênc. saúde coletiva vol.15 №.6 Rio de Janeiro Sept. p.1344-50.

14. Levay, P.F., M.Viljoen (1995) Lactoferrin: a general review // Haematologica, № 3, p. 252267.

15. Oppenheim J., J. Oppenheim, H. Fujiwara (1996) The role of cytokines in cancer // Cytokine Growth Factor Rev., Vol. 7. p. 279288.

16. Paradkar PH, Joshi JV, Mertia PN, Agashe SV, Vaidya RA.(2014) Role of cytokines in genesis, progression and prognosis of cervical cancer. //Asian Pac J Cancer Prev., №9, p.3851-64.

17. Shekari M, Kordi-Tamandani DM, MalekZadeh K, et al. (2012) Effect of anti-inflammatory (IL4, IL-10) cytokine genes in relation to risk of cervical carcinoma// Am J Clin Oncol, №35, p.514-9.

18. Storch J, Thumser AE.(2010) Tissue-specific functions in the fatty acid-binding protein family//J Biol Chem, , №285, p. 32679-83.

19. Yan Wang, Xiao-Hong Liu, Yue-Hong Li, and Ou Li (2013) The paradox of il-10-mediated modulation in cervical cancer// Biomed Rep. № 3, p. 347-351.

20. Zarogoulidis P, Yarmus L, Zarogoulidis K (2013). Interleukin-6 cytokine: a multifunctional glycoprotein for cancer. Immunome Res, 9, 35-40. 